\title{
Phentolamine and Cardiovascular Performance
}

\author{
LAWRENCE GOULD, MOHAMMAD ZAHIR, AND STEPHEN ETTINGER \\ with the technical assistance of Mary Di Lieto \\ From the Medical Service of the Bronx Veterans Administration Hospital, 130 West Kingsbridge Road, \\ Bronx, New York 10468, and the Animal Medical Center, New York City, New York, U.S.A.
}

Ahlquist (1948) proposed two distinct adrenergic receptor sites, the alpha- and beta-adrenergic receptors. Stimulation of the alpha-adrenergic receptors produces arteriolar constriction unaccompanied by any primary cardiac action, presumably due to the absence of alpha receptors in the heart. Selective blockade of the alpha receptors produces arteriolar dilatation, and this also is unaccompanied by any primary cardiac effect. This blockade can be accomplished by using the drug phentolamine.

Despite intensive studies on the adrenergic blocking activity of phentolamine there have been few reports analysing its haemodynamic effects and its mode of action. Taylor and his co-workers $(1965 \mathrm{a}, \mathrm{b}, \mathrm{c})$ have investigated the haemodynamic effects in man of the acute intravenous injection of $5 \mathrm{mg}$. phentolamine and of intravenous infusion of the drug at a rate of $2 \mathrm{mg}$. per minute for 10 minutes. Both methods of administration produced an increase in the heart rate and cardiac output and a decrease in the systemic arterial pressure. They concluded that the hypotensive effect limited the clinical applicability of the drug.

We now report an investigation of the action of phentolamine in the dog, in which a marked increase in the force of myocardial contraction was shown. Based on this finding, the study was extended to investigate more fully the haemodynamic actions of this drug in man.

\section{MethodS}

Animals. Four normal mongrel dogs weighing $11 \cdot 3$ kg. to $22 \cdot 7 \mathrm{~kg}$. $(25-50 \mathrm{lb}$.) were studied. They were lightly pre-anaesthetized with acetyl-promazine $(1 \mathrm{mg}$./ $11.3 \mathrm{~kg}$.) and succinyl-choline ( $1 \mathrm{mg} . / 2.7 \mathrm{~kg}$.). Pentothal was given intravenously until endotracheal intubation was established. They were maintained on a nitrous oxide and oxygen gas mixture, with intermittent

Received September 9, 1968. administration of methoxyflurane. Respiration was kept constant by a Bird respirator through a cuffed endotracheal tube.

After making an intercostal incision and spreading the ribs, the pericardium was opened and a strain gauge arch was attached on the right ventricle. Arterial blood pressure was measured through a Cournand needle in the femoral artery by means of a Statham pressure transducer. Continuous recordings of myocardial contractile force and blood pressure were made simultaneously with a multichannel oscillograph before and after the rapid intravenous injection of $5 \mathrm{mg}$. of phentolamine.

Human Subjects. Our observations were made in 5 patients (Group 1) with relatively normal left ventricles, who had never been in left ventricular failure, and 13 patients (Group 2) with abnormal left ventricles, who had previously been in left ventricular failure, and who underwent right and left heart catheterizations primarily as an aid to their clinical management. The diagnoses of the patients are listed in Table II.

Patients 4 and 16 were in atrial fibrillation; the rest were in sinus rhythm. Patients $3,4,6,8,9,13,14,15$, 16 , and 17 were taking digoxin; the rest were not receiving any therapy.

Catheterization of the left ventricle was performed either by the Seldinger technique or by retrograde arterial catheterization through a brachial arteriotomy. A Cournand needle was placed into the opposite brachial artery. In order to ensure high fidelity tracings, the needle was directly connected to a Statham pressure tranducer-P23Db. The first derivative of the brachial artery pressure could then be computed by an R-C differentiating circuit. A No. 7 Goodale-Lubin catheter was placed in the pulmonary artery. Cardiac output was determined by the Fick technique.

Left ventricular, pulmonary arterial, and systemic arterial pressures and cardiac output were measured in the resting state. The brachial artery $\mathrm{dp} / \mathrm{dt}$, and the left ventricular $\mathrm{dp} / \mathrm{dt}$, were also obtained. The latter was recorded through a catheter manometer system and measurements were made only when the pressure curves showed an undistorted wave form. Since the catheter values faithfully reproduce directional changes (Murphy 
et al., 1964), relative values of left ventricular $\mathrm{dp} / \mathrm{dt}$ are reported in this study; no attempt was made to quantitate these values in absolute terms. Two of the patients in Group 1, and 7 of the patients in Group 2, then pedalled a bicycle ergometer for 6 to 7 minutes in the supine position. Pressures and cardiac output were measured 5 minutes after the onset of exercise.

At least 20 minutes after exercise, when the pressure in the pulmonary artery as well as the pressures and first derivatives in the left ventricle and brachial artery had returned to the control values, phentolamine was given by constant intravenous drip ( $0.3 \mathrm{mg}$. per minute). Pressure recordings and cardiac output data were repeated 10 and 20 minutes after the start of infusion. In the patients not exercised, phentolamine was infused after the resting control values had been recorded.

In 10 patients in the resting state, the left ventricular end-diastolic volume was calculated cine-angiographically, using a method based on the uniplanar modification of the methods of Dodge et al. (1960), and Klein, Herman, and Gorlin (1967). Ventriculograms were taken in the $30^{\circ}$ right anterior oblique projection with a fixed distance from $x$-ray tube to table top. Cine films of a grid were made at the approximate level of the cardiac apex. The projected distance of this grid image was adjusted until actual and projected measurements were matched, so that left ventricular silhouettes free from the error of distortion and magnification were then obtained. The end-diastolic area (A) of the image was obtained by planimetry. The long axis (L) was measured directly. The width was calculated from the equation $W=\frac{4 A}{L \pi}$. The left ventricular end-diastolic volume was then obtained from the equation $L V E D V=4 / 3 \pi\left(\frac{W}{2}\right)^{2} \cdot \frac{L}{2}$. The end-systolic volume was calculated by subtracting the stroke volume obtained by the Fick technique from the left ventricular end-diastolic volume. No attempt was made to calculate the end-systolic volume angiographically, since the reliability of this measurement is in doubt (Davilla and Sanmarco, 1966). After completion of the 20-minute cardiac output determination, but while the phentolamine infusion was still running, left ventricular angiograms were performed in 10 subjects.

Left ventricular function was assessed according to the following parameters:

(1) $\mathrm{LVSW}=\frac{\mathrm{LV}_{\mathrm{s}}}{100}-\mathrm{LV}_{\mathrm{D}} \times 1.36 \times 1.050 \times \mathrm{SI}$

(2) $\mathrm{LVSP}=\mathrm{LVSW} / \mathrm{SEP}$
(3) $M S E R=S I / S E P$

(4) $\mathrm{TTI}_{\mathrm{B}}=\mathrm{LV}_{\mathrm{s}} \times \mathrm{SEP}$

(5) $\mathrm{TTI}_{\mathrm{m}}=\mathrm{LV}_{\mathrm{s}} \mathrm{SEP} \times \mathrm{HR}$

(6) $\mathrm{LVW}=\mathrm{CI} \times 1.36 \times 1.050 \times \mathrm{BA}_{\mathrm{m}} / 100$

(7) $\mathrm{PR}=\frac{\mathrm{BA}_{\mathrm{m}} \times 1332}{\mathrm{CO}}$

where $L V S W=$ left ventricular stroke work (g.m./beat/ $\mathrm{m}^{2} \quad \mathrm{BSA}$ ); SI = stroke index (ml./beat $/ \mathrm{m} .^{2}$ BSA); $\mathrm{LV}_{\mathrm{s}}=$ mean left ventricular systolic pressure $(\mathrm{mm} . \mathrm{Hg})$; $\mathrm{LV}_{\mathrm{D}}=$ left ventricular end-diastolic pressure (mm. $\left.\mathrm{Hg}\right)$; $L V S P=$ mean left ventricular stroke power (g.m./beat/ $\mathrm{m}^{2}$ BSA/systolic sec.); SEP=mean systolic ejection period (seconds); $M S E R=$ mean systolic ejection rate (ml./beat $/ \mathrm{m}^{2}{ }^{2} \mathrm{BSA} /$ systolic sec.); $\mathrm{TTI}_{\mathrm{B}}=$ tension time index per beat (mm. $\mathrm{Hg} \mathrm{sec}$./beat); $\mathrm{TTI}_{\mathrm{m}}=$ tension time index per minute ( $\mathrm{mm}$. $\mathrm{Hg}$ seconds/min.); $\mathrm{HR}=$ heart rate (beats/min.); LVW = left ventricular work (kg. m.l $\left.\mathrm{min} / \mathrm{m}^{2} \mathrm{BSA}\right) ; \mathbf{B A}_{\mathrm{m}}=$ mean brachial artery pressure (mm. $\mathrm{Hg}$ ); $\mathbf{P R}=$ systemic peripheral resistance (dynes/ sec. $/ \mathrm{cm}^{-5}$ ). CO=cardiac output (ml./sec.); $1.36=$ conversion factor from $\mathrm{mm}$. $\mathrm{Hg}$ to $\mathrm{cm} . \mathrm{H}_{2} \mathrm{O} ; 1 \cdot 050=$ whole blood specific gravity; $\mathrm{BSA}=$ body surface area in square meters.

\section{RESULTS}

Animal Studies. The effects of phentolamine on heart rate, arterial pressure, and right ventricular contractile force are shown in Table I. The amplitudes of the contractile force recordings were measured in millimeters.

The intravenous administration of phentolamine increased the ventricular contractile force in all 4 dogs (Fig.). There was an average increase of 102 per cent. The response to the drug was transient, lasting about 5 minutes. There was a variable response of the pulse rate and blood pressure.

Human Subjects. Complete data on all 18 patients are presented in Tables II and III. In addition, average values and the percentage change from the control value after exercise and phentolamine infusion are listed in Table IV.

Exercise. Only 2 patients in Group 1 were exercised. Therefore, no valid comparison can be

TABLE I

CARDIOVASCULAR EFFECTS OF PHENTOLAMINE IN THE DOG

\begin{tabular}{|c|c|c|c|c|c|c|c|c|c|}
\hline \multirow[t]{2}{*}{ Dog } & \multicolumn{3}{|c|}{ Control } & \multicolumn{3}{|c|}{ Peak effect of phentolamine } & \multicolumn{3}{|c|}{ Change } \\
\hline & $\begin{array}{c}\text { Cardiac } \\
\text { force } \\
\text { (mm.) }\end{array}$ & $\begin{array}{c}\text { Femoral } \\
\text { artery } \\
\text { (mm. Hg) }\end{array}$ & $\begin{array}{c}\text { Heart rate } \\
\text { (beats/min.) }\end{array}$ & $\begin{array}{c}\text { Cardiac } \\
\text { force } \\
\text { (mm.) }\end{array}$ & $\begin{array}{c}\text { Femoral } \\
\text { artery } \\
(\mathbf{m m} . \mathbf{H g})\end{array}$ & $\begin{array}{c}\text { Heart rate } \\
\text { (beats/min.) }\end{array}$ & $\begin{array}{c}\text { Cardiac } \\
\text { force } \\
(\%)\end{array}$ & $\begin{array}{c}\text { Femoral } \\
\text { artery } \\
(\mathrm{mm} . \mathbf{H g})\end{array}$ & $\begin{array}{c}\text { Heart rate } \\
\text { (beats/min.) }\end{array}$ \\
\hline $\begin{array}{l}1 \\
2 \\
3 \\
4\end{array}$ & $\begin{array}{r}2 \\
18 \\
4 \\
5\end{array}$ & $\begin{array}{l}115 / 80 \\
175 / 125 \\
120 / 90 \\
140 / 90\end{array}$ & $\begin{array}{l}120 \\
140 \\
180 \\
200\end{array}$ & $\begin{array}{r}6 \\
24 \\
7 \\
8\end{array}$ & $\begin{array}{c}110 / 70 \\
175 / 120 \\
75 / 50 \\
145 / 93\end{array}$ & $\begin{array}{l}160 \\
160 \\
180 \\
200\end{array}$ & $\begin{array}{c}200 \\
33 \frac{1}{3} \\
75 \\
100\end{array}$ & $\begin{aligned}-5 / & -10 \\
0 / & -5 \\
-45 / & -40 \\
5 & =-3\end{aligned}$ & $\begin{array}{r}+40 \\
+20 \\
0 \\
0\end{array}$ \\
\hline
\end{tabular}


TABLE IIA

COMPLETE OBSERVED HAEMODYNAMIC DATA ON 18 PATIENTS

\begin{tabular}{|c|c|c|c|c|c|c|c|c|c|c|c|c|c|}
\hline \multirow{2}{*}{$\begin{array}{c}\text { Patient } \\
\text { No. and } \\
\text { condition }\end{array}$} & \multirow{2}{*}{$\begin{array}{l}\text { Experim. } \\
\text { state }\end{array}$} & \multirow{2}{*}{$\begin{array}{c}\text { Rt. } \\
\text { atrium } \\
\text { mean } \\
(\underset{\mathbf{m m}}{\mathbf{H g}} \text {. }\end{array}$} & \multirow{2}{*}{$\begin{array}{l}\text { Rt. } \\
\text { ventr. } \\
\text { (mm. } \\
\text { Hg) }\end{array}$} & \multirow{2}{*}{ 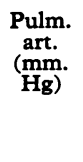 } & \multirow[t]{2}{*}{ Mean } & \multirow{2}{*}{$\begin{array}{c}\text { Lt. } \\
\text { ventr. } \\
\text { (mm. } \\
\mathbf{H g})\end{array}$} & \multirow{2}{*}{$\begin{array}{c}\text { Per } \\
\text { cent } \\
\text { lt.ventr. } \\
\mathrm{dp} / \mathrm{dt} \\
(\mathrm{mm} . \\
\mathrm{Hg} / \\
\text { sec.) }\end{array}$} & \multicolumn{2}{|c|}{ Brach. art. } & \multirow{2}{*}{$\begin{array}{c}\text { Brach. } \\
\text { art. } \\
\text { dp/dt } \\
\underset{\text { (mm. }}{\mathbf{H g} /} \\
\text { sec.) }\end{array}$} & \multirow{2}{*}{$\begin{array}{c}\text { Rate } \\
\text { (beats! } \\
\text { min.) }\end{array}$} & \multicolumn{2}{|c|}{$\begin{array}{c}\text { Syst. ejection } \\
\text { time }\end{array}$} \\
\hline & & & & & & & & $\stackrel{(\mathrm{mm}}{\mathbf{H g}}$. & Mean & & & $\begin{array}{l}\text { Sec./ } \\
\text { beat }\end{array}$ & $\begin{array}{c}\text { Rate } \\
\text { correct }\end{array}$ \\
\hline $\begin{array}{l}1 \\
\text { Normal } \\
\text { functional } \\
\text { murmur } \\
2 \\
\text { Mitral } \\
\text { stenosis } \\
3 \\
\text { Mitral } \\
\text { stenosis } \\
4 \\
\text { Mitral } \\
\text { stenosis } \\
5 \\
\text { Idiopathic } \\
\text { hypertrophic } \\
\text { subaortic } \\
\text { stenosis }\end{array}$ & $\left\{\begin{array}{l}\text { Rest } \\
\text { Exercise } \\
\text { Phentol. } \\
\text { Phentol. } \\
\text { Rest } \\
\text { Phentol. } \\
\text { Phentol. } \\
\text { Pest } \\
\text { Phentol. } \\
\text { Phentol. } \\
\text { Pest } \\
\text { Phentol. } \\
\text { Phentol. } \\
\text { Rest } \\
\text { Exercise } \\
\text { Phentol. } \\
\text { Phentol. }\end{array}\right.$ & 2 & $\begin{array}{l}45 / 5 \\
50 / 4\end{array}$ & $\begin{array}{l}20 / 8 \\
34 / 10 \\
\\
70 / 28 \\
60 / 30 \\
60 / 30 \\
45 / 18 \\
50 / 25 \\
50 / 10 \\
55 / 22 \\
55 / 30 \\
20 / 9 \\
35 / 15 \\
20 / 9 \\
22 / 9\end{array}$ & $\begin{array}{l}45 \\
40 \\
40 \\
30 \\
35 \\
22 \\
35 \\
\\
38 \\
12 \\
20 \\
12 \\
13\end{array}$ & $\begin{array}{l}140 / 9 \\
160 / 9 \\
135 / 4 \\
130 / 4 \\
130 / 3 \\
150 / 5 \\
145 / 3 \\
90 / 8 \\
100 / 10 \\
95 / 10 \\
125 / 5 \\
130 / 5 \\
125 / 5 \\
130 / 0 \\
145 / 12 \\
135 / 0 \\
135 / 0\end{array}$ & $\begin{array}{l}+64 \\
+64 \\
+91 \\
+18 \\
+14 \\
+68 \\
+76 \\
+27 \\
+64 \\
+50 \\
+62 \\
+44\end{array}$ & $\begin{array}{l}140 / 80 \\
160 / 90 \\
140 / 90 \\
140 / 90 \\
130 / 80 \\
160 / 90 \\
150 / 80 \\
100 / 60 \\
110 / 65 \\
100 / 65 \\
140 / 90 \\
130 / 80 \\
125 / 85 \\
120 / 80 \\
110 / 60 \\
135 / 75 \\
110 / 70\end{array}$ & $\begin{array}{r}95 \\
115 \\
110 \\
110 \\
95 \\
115 \\
100 \\
75 \\
80 \\
77 \\
110 \\
95 \\
100 \\
92 \\
80 \\
95 \\
85\end{array}$ & $\begin{array}{l}680 \\
950 \\
820 \\
850 \\
543 \\
755 \\
725 \\
750 \\
685 \\
650 \\
490 \\
630 \\
630 \\
700 \\
880 \\
880\end{array}$ & $\begin{array}{r}65 \\
88 \\
88 \\
88 \\
60 \\
65 \\
65 \\
58 \\
58 \\
62 \\
100 \\
100 \\
110 \\
72 \\
88 \\
79 \\
75\end{array}$ & $\begin{array}{l}0 \cdot 28 \\
0.32 \\
0 \cdot 30 \\
0 \cdot 28 \\
0 \cdot 30 \\
0 \cdot 29 \\
0 \cdot 29 \\
0 \cdot 28 \\
0 \cdot 30 \\
0 \cdot 30 \\
0 \cdot 24 \\
0 \cdot 24 \\
0 \cdot 22 \\
0.30 \\
0.30 \\
0.28 \\
0.28\end{array}$ & $\begin{array}{l}0.38 \\
0.46 \\
0.44 \\
0.42 \\
0.40 \\
0.39 \\
0.39 \\
0.38 \\
0.40 \\
0.40 \\
0.40 \\
0.40 \\
0.40 \\
0.42 \\
0.44 \\
0.41 \\
0.40\end{array}$ \\
\hline $\begin{array}{l}\text { stenosis } \\
6 \\
\text { Primary } \\
\text { myocardial } \\
\text { disease } \\
\mathbf{7} \\
\text { Primary } \\
\text { myocardial } \\
\text { disease } \\
\mathbf{8} \\
\text { Primary } \\
\text { myocardial } \\
\text { disease } \\
9 \\
\text { Primary } \\
\text { myocardial } \\
\text { disease } \\
\text { 10 } \\
\text { Primary } \\
\text { myocardial } \\
\text { disease }\end{array}$ & $\left\{\begin{array}{l}\text { Rest } \\
\text { Exercise } \\
\text { Phentol. } \\
\text { Phentol. } \\
\text { Rest } \\
\text { Exercise } \\
\text { Phentol. } \\
\text { Phentol. } \\
\text { Rest } \\
\text { Exercise } \\
\text { Phentol. } \\
\text { Phentol. } \\
\text { Rest } \\
\text { Exercise } \\
\text { Phentol. } \\
\text { Phentol. } \\
\text { Rest } \\
\text { Phentol. } \\
\text { Phentol. }\end{array}\right.$ & 0 & $20 / 2$ & $\begin{array}{c}32 / 11 \\
100 / 50 \\
35 / 12 \\
30 / 12 \\
45 / 20 \\
75 / 40 \\
50 / 35 \\
18 / 7 \\
45 / 17 \\
20 / 8 \\
22 / 8 \\
35 / 10 \\
70 / 30 \\
29 / 8 \\
32 / 10 \\
65 / 32 \\
80 / 35 \\
60 / 30\end{array}$ & $\begin{array}{l}18 \\
60 \\
20 \\
18 \\
30 \\
60 \\
\\
42 \\
11 \\
25 \\
12 \\
12 \\
18 \\
50 \\
13 \\
16 \\
45 \\
50 \\
40\end{array}$ & $\begin{array}{l}105 / 8 \\
175 / 30 \\
130 / 5 \\
120 / 0 \\
95 / 25 \\
115 / 28 \\
110 / 25 \\
110 / 25 \\
170 / 10 \\
180 / 20 \\
150 / 0 \\
150 / 0 \\
170 / 4 \\
200 / 15 \\
170 / 0 \\
200 / 0 \\
90 / 26 \\
95 / 26 \\
100 / 27\end{array}$ & $\begin{array}{r}+91 \\
+39 \\
+28 \\
+86 \\
+93 \\
+93 \\
+37 \\
+16 \\
+25 \\
+87 \\
+18 \\
+129 \\
+27 \\
+32\end{array}$ & $\begin{array}{l}110 / 80 \\
175 / 100 \\
145 / 100 \\
120 / 80 \\
100 / / 0 \\
120 / 100 \\
115 / 90 \\
140 / 95 \\
170 / 85 \\
180 / 90 \\
155 / 85 \\
170 / 100 \\
170 / 85 \\
210 / 110 \\
170 / 95 \\
200 / 100 \\
100 / 70 \\
110 / 80 \\
105 / 65\end{array}$ & $\begin{array}{r}90 \\
125 \\
115 \\
95 \\
88 \\
108 \\
100 \\
110 \\
115 \\
120 \\
110 \\
125 \\
115 \\
145 \\
120 \\
135 \\
80 \\
90 \\
80\end{array}$ & $\begin{array}{r}440 \\
630 \\
475 \\
475 \\
370 \\
616 \\
310 \\
780 \\
785 \\
1120 \\
1090 \\
1120 \\
1100 \\
1740 \\
1100 \\
1320 \\
425 \\
480 \\
425\end{array}$ & $\begin{array}{r}84 \\
100 \\
88 \\
88 \\
94 \\
125 \\
107 \\
115 \\
84 \\
100 \\
88 \\
100 \\
68 \\
100 \\
79 \\
88 \\
107 \\
115 \\
107\end{array}$ & $\begin{array}{l}0.24 \\
0.24 \\
0.24 \\
0.24 \\
0.20 \\
0.18 \\
0.20 \\
0.20 \\
0.28 \\
0.30 \\
0.30 \\
0.23 \\
0 \cdot 28 \\
0.30 \\
0.30 \\
0.28 \\
0.24 \\
0.22 \\
0.24\end{array}$ & $\begin{array}{l}0.37 \\
0.40 \\
0.38 \\
0.38 \\
0.35 \\
0.38 \\
0.37 \\
0.38 \\
0.41 \\
0.46 \\
0.44 \\
0.39 \\
0.38 \\
0.46 \\
0.43 \\
0.42 \\
0.41 \\
0.40 \\
0.41\end{array}$ \\
\hline $\begin{array}{l}\text { d1 } \\
\text { Primary } \\
\text { myocardial } \\
\text { disease } \\
12 \\
\text { Hypertension } \\
13 \\
\text { Aortic } \\
\text { insufficiency } \\
14 \\
\text { Primary } \\
\text { myocardial }\end{array}$ & $\left\{\begin{array}{l}\text { Rest } \\
\text { Exercise } \\
\text { Phentol. } \\
\text { Phentol. }\end{array}\right.$ & 3 & $32 / 0$ & $\begin{array}{l}40 / 15 \\
90 / 40 \\
\\
26 / 11 \\
32 / 12 \\
70 / 35 \\
26 / 9 \\
24 / 7 \\
55 / 25 \\
75 / 40 \\
65 / 30 \\
60 / 35 \\
23 / 9 \\
16 / 7 \\
16 / 7\end{array}$ & $\begin{array}{l}22 \\
60 \\
\\
16 \\
20 \\
45 \\
15 \\
14 \\
35 \\
52 \\
40 \\
45 \\
14 \\
10 \\
10\end{array}$ & $\begin{array}{l}110 / 23 \\
150 / 30 \\
140 / 16 \\
115 / 2 \\
185 / 8 \\
220 / 20 \\
180 / 5 \\
175 / 0 \\
110 / 22 \\
130 / 30 \\
120 / 20 \\
120 / 16\end{array}$ & $\begin{array}{r}+94 \\
+30 \\
+20 \\
+55 \\
+33 \\
+55 \\
+118 \\
+51 \\
+66\end{array}$ & $\begin{array}{l}110 / 80 \\
150 / 100 \\
140 / 80 \\
115 / 75 \\
190 / 115 \\
220 / 90 \\
185 / 90 \\
190 / 95 \\
110 / 50 \\
140 / 65 \\
130 / 65 \\
130 / 55 \\
150 / 95 \\
160 / 90 \\
160 / 95\end{array}$ & $\begin{array}{r}90 \\
115 \\
100 \\
90 \\
140 \\
130 \\
120 \\
125 \\
70 \\
90 \\
85 \\
80 \\
113 \\
113 \\
116\end{array}$ & $\begin{array}{r}450 \\
\\
645 \\
310 \\
955 \\
1400 \\
1090 \\
1065 \\
1040 \\
1400 \\
1120 \\
1260 \\
660 \\
800 \\
820\end{array}$ & $\begin{array}{r}84 \\
115 \\
94 \\
94 \\
88 \\
115 \\
94 \\
107 \\
75 \\
107 \\
84 \\
88 \\
87 \\
107 \\
115\end{array}$ & $\begin{array}{l}0.20 \\
0.23 \\
0.24 \\
0.24 \\
0.24 \\
0.26 \\
0.24 \\
0.23 \\
0.30 \\
0.30 \\
0.30 \\
0.30 \\
0.23 \\
0.22 \\
0.20\end{array}$ & $\begin{array}{l}0.34 \\
0.41 \\
0.39 \\
0.39 \\
0.38 \\
0.44 \\
0.39 \\
0.40 \\
0.42 \\
0.47 \\
0.43 \\
0.44 \\
0.37 \\
0.39 \\
0.38\end{array}$ \\
\hline $\begin{array}{l}\text { disease } \\
15 \\
\text { Primary } \\
\text { myocardial } \\
\text { disease }\end{array}$ & $\left\{\begin{array}{l}\text { Rest } \\
\text { Phentol. } \\
\text { Phentol. }\end{array}\right.$ & 10 & $85 / 10$ & $\begin{array}{l}85 / 42 \\
75 / 40 \\
75 / 40\end{array}$ & $\begin{array}{l}56 \\
52 \\
52\end{array}$ & $\begin{array}{l}155 / 30 \\
16:) / 17 \\
150 / 17\end{array}$ & $\begin{array}{r}+14 \\
+9\end{array}$ & $\begin{array}{l}165 / 110 \\
170 / 105 \\
170 / 100\end{array}$ & $\begin{array}{l}128 \\
127 \\
124\end{array}$ & $\begin{array}{l}730 \\
850 \\
910\end{array}$ & $\begin{array}{l}115 \\
120 \\
115\end{array}$ & $\begin{array}{l}0.22 \\
0.22 \\
0.22\end{array}$ & $\begin{array}{l}0.40 \\
0.41 \\
0.40\end{array}$ \\
\hline $\begin{array}{l}16 \\
16 \\
\text { Aortic } \\
\text { insufficiency } \\
17 \\
\text { Aortic } \\
\text { stenosis } \\
18 \\
\text { Carcinoid, } \\
\text { myocardial } \\
\text { and tricuspid } \\
\text { incomp., } \\
\text { pulm. stenosis }\end{array}$ & $\left\{\begin{array}{l}\text { Rest } \\
\text { Phentol. } \\
\text { Phentol. } \\
\text { Rest } \\
\text { Phentol. } \\
\text { Phentol. } \\
\text { Rest } \\
\text { Phentol. } \\
\text { Phentol. }\end{array}\right.$ & 8 & $\begin{array}{l}30 / 0 \\
29 / 2 \\
30 / 8\end{array}$ & $\begin{array}{l}30 / 10 \\
27 / 8 \\
24 / 8 \\
28 / 13 \\
22 / 10 \\
22 / 10 \\
20 / 10 \\
20 / 6 \\
18 / 6\end{array}$ & $\begin{array}{l}17 \\
14 \\
13 \\
18 \\
14 \\
14 \\
14 \\
11 \\
10\end{array}$ & $\begin{array}{l}135 / 8 \\
130 / 8 \\
130 / 7 \\
175 / 5 \\
180 / 5 \\
175 / 5 \\
135 / 3 \\
150 / 2 \\
158 / 2\end{array}$ & $\begin{array}{l}+11 \\
+11 \\
+24 \\
+33 \\
+23 \\
+23\end{array}$ & $\begin{array}{l}138 / 53 \\
150 / 52 \\
142 / 62 \\
150 / 85 \\
160 / 75 \\
160 / 85 \\
155 / 90 \\
165 / 85 \\
165 / 85\end{array}$ & $\begin{array}{r}81 \\
85 \\
88 \\
106 \\
103 \\
110 \\
112 \\
112 \\
112\end{array}$ & $\begin{array}{r}930 \\
1080 \\
1120 \\
480 \\
630 \\
610 \\
850 \\
1000 \\
1020\end{array}$ & $\begin{array}{l}60 \\
46 \\
46 \\
63 \\
65 \\
68 \\
83 \\
88 \\
88\end{array}$ & $\begin{array}{l}0.28 \\
0.26 \\
0.26 \\
0.38 \\
0.34 \\
0.34 \\
0.30 \\
0.28 \\
0.28\end{array}$ & $\begin{array}{l}0.38 \\
0.33 \\
0.33 \\
0.48 \\
0.44 \\
0.44 \\
0.43 \\
0.42 \\
0.42\end{array}$ \\
\hline
\end{tabular}

made with the control state. In Group 2 comparison is possible, since 7 of the 13 patients were exercised. During the stress of exercise, the oxygen consumption increased by 187 per cent and the arteriovenous oxygen difference widened by 74 per cent. The cardiac index rose 64 per cent; this was associated with a 24 per cent fall in the peripheral resistance. The rise in the cardiac index 
Phentolamine and Cardiovascular Performance

TABLE IIB

COMPLETE DERIVED HAEMODYNAMIC DATA ON 18 PATIENTS

\begin{tabular}{|c|c|c|c|c|c|c|c|c|c|c|c|c|}
\hline $\begin{array}{l}\text { Patient } \\
\text { No. and } \\
\text { condition }\end{array}$ & $\begin{array}{c}\text { Experim. } \\
\text { state }\end{array}$ & $\begin{array}{c}\mathrm{AV} \\
\mathrm{O}_{2} \\
\text { diff. } \\
\text { (vol. \%) }\end{array}$ & $\begin{array}{c}\mathrm{O}_{2} \text { con. } \\
\text { sumpt. } \\
\text { (ml. } . / \\
\text { min. } . / \\
\text { m. }^{2} \text { ) }\end{array}$ & $\begin{array}{c}\text { Cardiac } \\
\text { index } \\
(1.1 \\
\text { min. } \\
\text { m.2) }\end{array}$ & $\begin{array}{c}\text { Stroke } \\
\text { index } \\
\text { (ml./ } \\
\text { beat } \\
\left.\text { m. }{ }^{2}\right)\end{array}$ & \begin{tabular}{|c|} 
LVSWWI \\
(g.m./ \\
beat/ \\
m.2)
\end{tabular} & $\begin{array}{c}\text { LVSP } \\
\text { (g.m././.2/ } \\
\text { beat.'m.2/ } \\
\text { syst. sec.) }\end{array}$ & $\begin{array}{c}\text { MSER } \\
\text { (ml./beat/ } \\
\text { m.//syst. } \\
\text { sec.) }\end{array}$ & $\begin{array}{l}\text { TTI }_{\mathrm{b}} \\
\text { (mm. } \\
\mathbf{H g} \mid \\
\text { sec./ } \\
\text { beat) }\end{array}$ & $\begin{array}{c}\operatorname{TTI}_{\mathbf{m}} \\
\text { (mm. } \\
\mathbf{H g} \mid \\
\text { sec. } \mid \\
\text { min. })\end{array}$ & $\begin{array}{c}\text { Lt. } \\
\text { ventr. } \\
\text { work } \\
\text { (kg.m. } \\
\text { min. } / \\
\text { min. })^{2} \\
\text { m. }\end{array}$ & $\begin{array}{l}\text { Periph. } \\
\text { resist. } \\
\text { (dyne } \\
\text { sec.- } \\
\text { cm. }-5 \text { ) }\end{array}$ \\
\hline \multirow{8}{*}{ 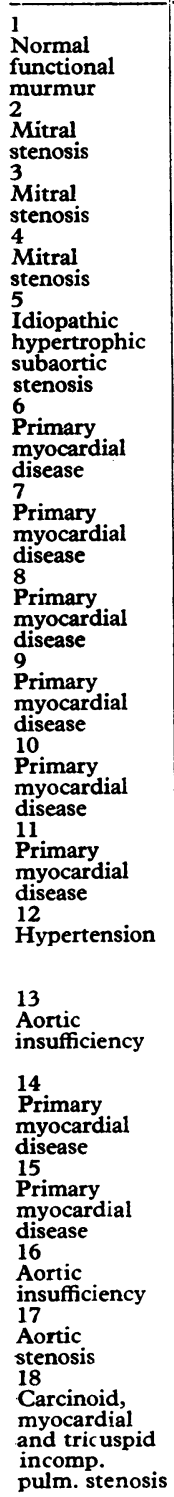 } & $\left\{\begin{array}{l}\text { Rest } \\
\text { Exercise } \\
\text { Phentol. } \\
\text { Phentol. } \\
\text { Rest } \\
\text { Phentol. } \\
\text { Phentol. } \\
\text { Rest } \\
\text { Phentol. } \\
\text { Phentol. } \\
\text { Rest } \\
\text { Phentol. } \\
\text { Phentol. } \\
\text { Rest } \\
\text { Exercise } \\
\text { Phentol. } \\
\text { Phentol. }\end{array}\right.$ & $\begin{array}{l}3.6 \\
4.8 \\
2.5 \\
2.9 \\
4.2 \\
5.6 \\
5.5 \\
4.5 \\
5.4 \\
3.6 \\
5.7 \\
4.7 \\
5.3 \\
3.4 \\
5.8 \\
2.5 \\
4.4\end{array}$ & $\begin{array}{r}94 \\
265 \\
95 \\
107 \\
167 \\
148 \\
172 \\
131 \\
148 \\
139 \\
145 \\
102 \\
101 \\
148 \\
287 \\
177 \\
147\end{array}$ & $\begin{array}{l}2 \cdot 6 \\
5 \cdot 52 \\
3 \cdot 77 \\
3 \cdot 70 \\
4 \cdot 0 \\
2 \cdot 66 \\
3 \cdot 13 \\
2 \cdot 91 \\
2 \cdot 73 \\
3 \cdot 89 \\
2 \cdot 48 \\
2 \cdot 15 \\
1 \cdot 91 \\
4 \cdot 36 \\
4 \cdot 94 \\
7 \cdot 1 \\
3 \cdot 35\end{array}$ & $\begin{array}{l}40 \\
63 \\
42 \\
42 \\
67 \\
41 \\
48 \\
50 \\
47 \\
62 \\
25 \\
22 \\
18 \\
60 \\
56 \\
90 \\
44\end{array}$ & $\begin{array}{r}75 \\
136 \\
82 \\
76 \\
122 \\
85 \\
98 \\
59 \\
61 \\
75 \\
43 \\
39 \\
31 \\
112 \\
107 \\
174 \\
85\end{array}$ & $\begin{array}{l}269 \\
424 \\
273 \\
272 \\
407 \\
293 \\
338 \\
210 \\
203 \\
250 \\
178 \\
163 \\
141 \\
373 \\
357 \\
620 \\
302\end{array}$ & $\begin{array}{r}143 \\
192 \\
140 \\
150 \\
223 \\
142 \\
165 \\
179 \\
157 \\
207 \\
104 \\
92 \\
82 \\
200 \\
187 \\
322 \\
157\end{array}$ & $\begin{array}{l}39 \\
51 \\
42 \\
37 \\
39 \\
44 \\
42 \\
25 \\
30 \\
29 \\
30 \\
31 \\
30 \\
39 \\
44 \\
38 \\
38\end{array}$ & $\begin{array}{l}2545 \\
4500 \\
3700 \\
3200 \\
2340 \\
2830 \\
2730 \\
1465 \\
1740 \\
1770 \\
3000 \\
3120 \\
3020 \\
2810 \\
3820 \\
3000 \\
2840\end{array}$ & $\begin{array}{l}3.5 \\
9.1 \\
5.9 \\
5.8 \\
5.4 \\
4.4 \\
4.5 \\
3.1 \\
3.1 \\
4.3 \\
3.9 \\
2.9 \\
2.7 \\
5.7 \\
5.7 \\
9.7 \\
4.1\end{array}$ & $\begin{array}{r}1490 \\
853 \\
1190 \\
1201 \\
1100 \\
2010 \\
1570 \\
1120 \\
1280 \\
870 \\
1730 \\
1690 \\
2900 \\
886 \\
680 \\
565 \\
1070\end{array}$ \\
\hline & 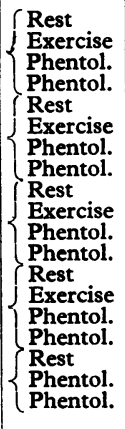 & $\begin{array}{r}5.0 \\
10.1 \\
3.7 \\
3.4 \\
8.4 \\
12.5 \\
6.9 \\
6.5 \\
4.4 \\
8.4 \\
5.7 \\
4.8 \\
4.3 \\
6.9 \\
4.3 \\
5.6 \\
7.0 \\
5.1 \\
4.3\end{array}$ & $\begin{array}{l}133 \\
329 \\
137 \\
121 \\
130 \\
234 \\
134 \\
147 \\
110 \\
246 \\
152 \\
136 \\
96 \\
406 \\
128 \\
193 \\
1165 \\
202 \\
153\end{array}$ & $\begin{array}{l}2 \cdot 64 \\
3 \cdot 25 \\
3 \cdot 70 \\
3.58 \\
1.54 \\
1.87 \\
1.93 \\
2 \cdot 26 \\
2 \cdot 50 \\
3 \cdot 08 \\
2 \cdot 67 \\
2 \cdot 82 \\
2 \cdot 22 \\
5 \cdot 52 \\
2.97 \\
3.44 \\
2 \cdot 36 \\
3.96 \\
3.59\end{array}$ & $\begin{array}{l}31 \\
33 \\
43 \\
41 \\
16 \\
16 \\
18 \\
20 \\
29 \\
31 \\
31 \\
28 \\
33 \\
59 \\
38 \\
39 \\
22 \\
35 \\
34\end{array}$ & $\begin{array}{c}43 \\
68 \\
77 \\
70 \\
16 \\
20 \\
22 \\
25 \\
66 \\
71 \\
67 \\
60 \\
78 \\
156 \\
92 \\
111 \\
20 \\
34 \\
35\end{array}$ & $\begin{array}{l}179 \\
284 \\
320 \\
292 \\
80 \\
111 \\
110 \\
125 \\
236 \\
237 \\
223 \\
260 \\
278 \\
520 \\
307 \\
396 \\
83 \\
155 \\
146\end{array}$ & $\begin{array}{r}129 \\
137 \\
179 \\
171 \\
80 \\
89 \\
90 \\
100 \\
104 \\
103 \\
103 \\
122 \\
118 \\
263 \\
127 \\
139 \\
92 \\
159 \\
142\end{array}$ & $\begin{array}{l}25 \\
42 \\
31 \\
29 \\
19 \\
21 \\
22 \\
22 \\
48 \\
54 \\
45 \\
35 \\
48 \\
60 \\
51 \\
56 \\
22 \\
21 \\
24\end{array}$ & $\begin{array}{l}2120 \\
4200 \\
2750 \\
2540 \\
1785 \\
2590 \\
2350 \\
2530 \\
4000 \\
5400 \\
3980 \\
3450 \\
3230 \\
6000 \\
4030 \\
4940 \\
2310 \\
2300 \\
2570\end{array}$ & $\begin{array}{r}3 \cdot 4 \\
5.5 \\
6.1 \\
4.9 \\
1.9 \\
2.9 \\
2 \cdot 8 \\
3.5 \\
4 \cdot 1 \\
5 \cdot 3 \\
4 \cdot 2 \\
5.0 \\
3.7 \\
11.4 \\
5.1 \\
6 \cdot 6 \\
2.7 \\
5.1 \\
4 \cdot 1\end{array}$ & $\begin{array}{l}1540 \\
1700 \\
1365 \\
1170 \\
2260 \\
2280 \\
2020 \\
1930 \\
2010 \\
1780 \\
1810 \\
1930 \\
2040 \\
970 \\
1585 \\
1550 \\
1625 \\
1210 \\
1065\end{array}$ \\
\hline & \multirow{2}{*}{ 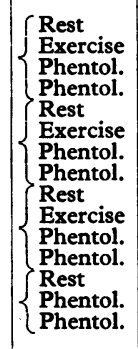 } & $\begin{array}{l}6 \cdot 1 \\
9 \cdot 9 \\
5 \cdot 4\end{array}$ & $\begin{array}{l}117 \\
484 \\
124\end{array}$ & $\begin{array}{l}1.91 \\
4.9 \\
2 \cdot 29\end{array}$ & $\begin{array}{l}22 \\
43 \\
24\end{array}$ & $\begin{array}{l}27 \\
74 \\
43\end{array}$ & & & \multirow{2}{*}{$\begin{array}{l}22 \\
35 \\
34 \\
28 \\
44 \\
57 \\
43 \\
40 \\
33 \\
39 \\
36 \\
36\end{array}$} & \multirow{2}{*}{$\begin{array}{l}1850 \\
3970 \\
3160 \\
2600 \\
3900 \\
6560 \\
4060 \\
4300 \\
2480 \\
4160 \\
3300 \\
3170\end{array}$} & & $\begin{array}{r}1870 \\
930 \\
1730\end{array}$ \\
\hline & & $\begin{array}{l}4 \cdot 5 \\
9 \cdot 1 \\
4 \cdot 5 \\
4 \cdot 9 \\
5 \cdot 3 \\
9 \cdot 5 \\
5 \cdot 1 \\
3 \cdot 8 \\
4 \cdot 2 \\
4 \cdot 8 \\
4 \cdot 9\end{array}$ & $\begin{array}{l}114 \\
425 \\
138 \\
159 \\
158 \\
346 \\
1722 \\
1183 \\
1142 \\
165\end{array}$ & $\begin{array}{l}2.54 \\
4.58 \\
3.08 \\
3.26 \\
2.98 \\
3.63 \\
3.37 \\
4.80 \\
2.95 \\
2.96 \\
3.38\end{array}$ & $\begin{array}{l}29 \\
41 \\
33 \\
30 \\
40 \\
34 \\
40 \\
54 \\
34 \\
28 \\
29\end{array}$ & $\begin{array}{r}74 \\
117 \\
83 \\
75 \\
50 \\
49 \\
57 \\
80\end{array}$ & $\begin{array}{l}308 \\
450 \\
346 \\
326 \\
167 \\
163 \\
190 \\
267\end{array}$ & $\begin{array}{l}121 \\
158 \\
138 \\
130 \\
113 \\
1133 \\
1180 \\
148 \\
127 \\
145\end{array}$ & & & $\begin{array}{l}5.1 \\
8.5 \\
5.3 \\
5.8 \\
3.0 \\
4.7 \\
4.1 \\
5.5 \\
4.8 \\
4.8 \\
5.6\end{array}$ & $\begin{array}{l}2420 \\
1220 \\
1720 \\
1680 \\
990 \\
1050 \\
1070 \\
700 \\
2060 \\
2040 \\
1840\end{array}$ \\
\hline & \multirow{4}{*}{ 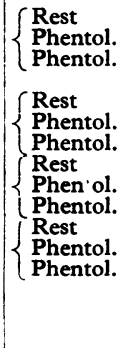 } & 4.5 & 190 & 2.73 & 24 & 43 & 196 & 109 & \multirow{4}{*}{$\begin{array}{l}34 \\
35 \\
33 \\
38 \\
34 \\
34 \\
66 \\
61 \\
60 \\
40 \\
42\end{array}$} & $\begin{array}{l}3920 \\
4202 \\
3800\end{array}$ & 5.0 & 1810 \\
\hline & & 6.7 & 153 & $2 \cdot 28$ & 38 & 69 & 247 & 136 & & 2270 & 2.6 & 1280 \\
\hline & & $\begin{array}{l}5 \cdot 7 \\
5 \cdot 9 \\
3 \cdot 4 \\
4 \cdot 3 \\
4 \cdot 6\end{array}$ & $\begin{array}{l}226 \\
145 \\
154 \\
178 \\
138\end{array}$ & $\begin{array}{l}3.98 \\
2 \cdot 46 \\
4 \cdot 54 \\
4 \cdot 16 \\
3 \cdot 0\end{array}$ & $\begin{array}{l}88 \\
39 \\
70 \\
61 \\
36\end{array}$ & $\begin{array}{r}155 \\
95 \\
175 \\
148 \\
68\end{array}$ & $\begin{array}{l}596 \\
250 \\
515 \\
435 \\
227\end{array}$ & $\begin{array}{l}338 \\
103 \\
206 \\
180 \\
120\end{array}$ & & $\begin{array}{l}1555 \\
4180 \\
3990 \\
4050 \\
3360\end{array}$ & $\begin{array}{l}5.0 \\
3.7 \\
6.7 \\
6.5 \\
4.8\end{array}$ & $\begin{array}{r}803 \\
1815 \\
965 \\
1125 \\
1770\end{array}$ \\
\hline & & $4 \cdot 8$ & 145 & $3 \cdot 24$ & 37 & 83 & 297 & 132 & & $\begin{array}{l}3700 \\
3890\end{array}$ & $5 \cdot 2$ & 1640 \\
\hline
\end{tabular}

LVSWI, left ventricular stroke work index; LVSP, left ventricular stroke power; MSER, mean systolic ejection rate; TTIb, tension time index per beat; TTIm, tension time index per minute.

was due to the 33 per cent increase in the heart rate and the 28 per cent increase in the stroke index. The left ventricular stroke work, left ventricular stroke power, and mean systolic ejection rate increased mainly because of the increase in the stroke index. The left ventricular $\mathrm{dp} / \mathrm{dt}$ rose 81 per cent 


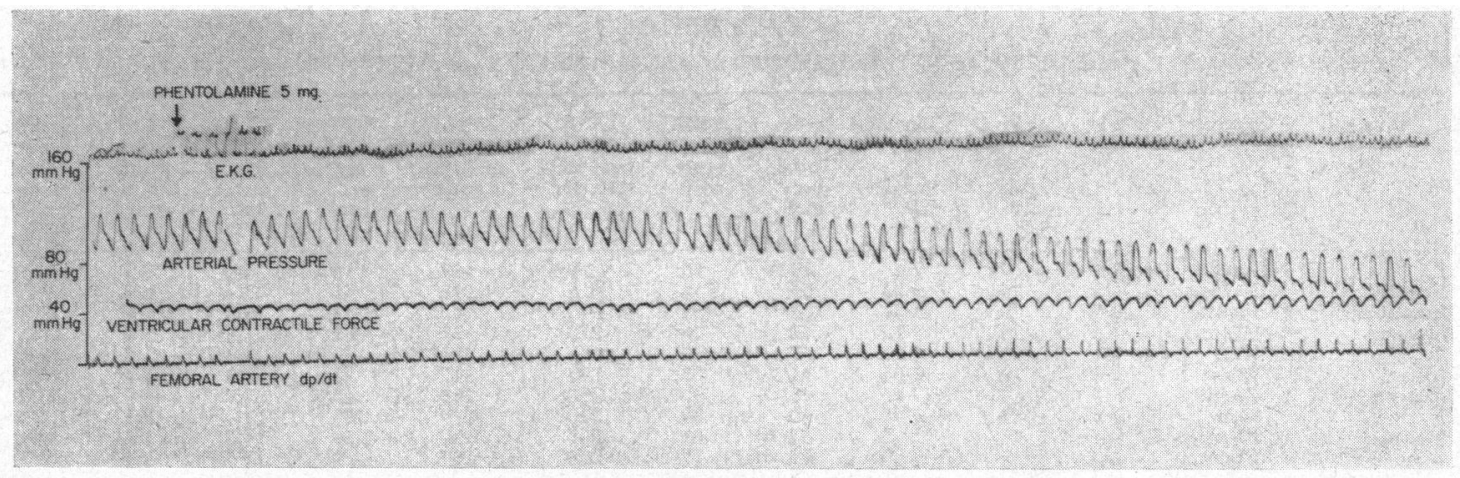

FIG.-The effects of $5 \mathrm{mg}$. phentolamine on right ventricular contractile force in a normal dog.

TABLE III

VENTRICULAR VOLUMES

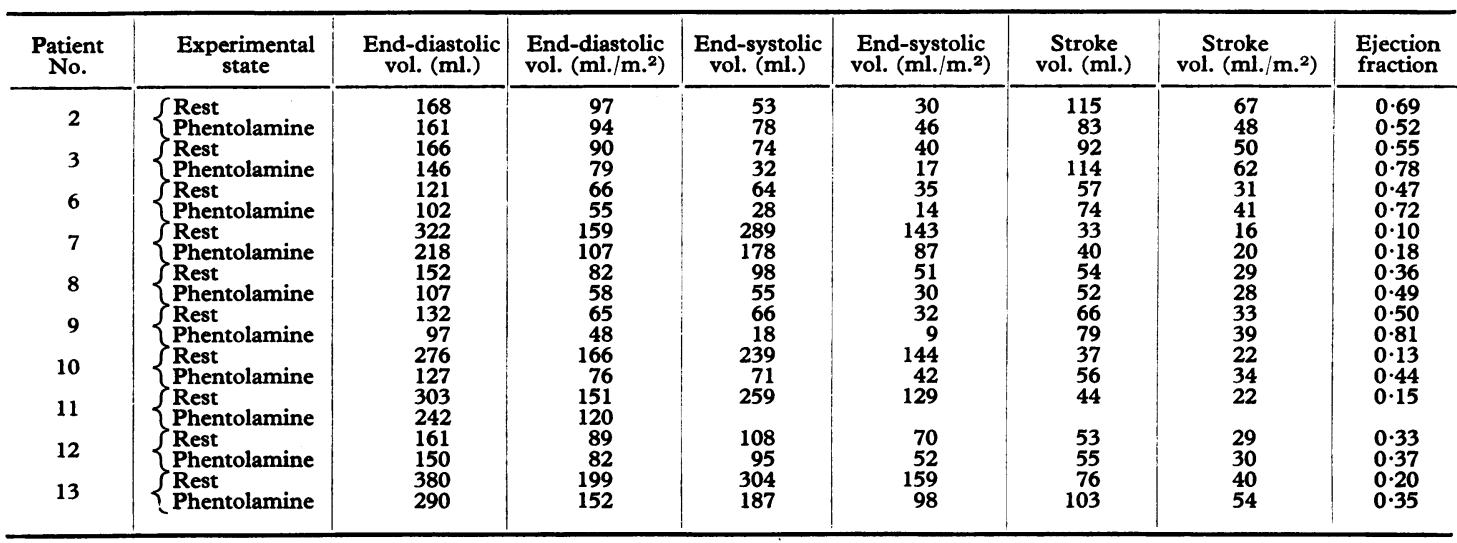

presumably because of an increase in left ventricular contractility. The left ventricular end-diastolic pressure rose 79 per cent with exercise.

Phentolamine. Among the subjects in Group 1, phentolamine produced minimal varying directional responses in the cardiac index and stroke index. The percentage change from the control value was negligible. The left ventricular $\mathrm{dp} / \mathrm{dt}$ rose 48 and 58 per cent at 10 and 20 minutes, respectively, due presumably to an increase in left ventricular contractility. There was only a minimal change in the brachial artery systolic pressure and pulse rate. Patient 5, with idiopathic hypertrophic subaortic stenosis, was of interest in that he first showed a significant increase followed by a decrease in the cardiac index. The late fall in output was associated with a larger systolic gradient, as measured between the left ventricle and brachial artery.

Among the subjects in Group 2, there were similar directional changes in the haemodynamic para- meters at the 10- and 20-minute test period. In contrast to exercise, phentolamine administration after 20 minutes produced only a 23 per cent increase in oxygen consumption, but the arteriovenous oxygen difference narrowed by 14 per cent. (When the AV difference was less than 5 vol. $\%$ it increased with phentolamine, and when it was more than 5 vol. $\%$ it decreased.) A marked increase in the cardiac index occurred, which was associated with a 24 per cent reduction in the peripheral resistance. In patients 6,7 , and 13 , the cardiac index rose more with phentolamine than with exercise. The rise in the cardiac index was not only due to the 12 per cent rate increase, but also to the 35 per cent rise in the stroke index. An increase in the mean systolic ejection rate, left ventricular stroke power, and left ventricular stroke work occurred, as would be expected with an increase in the stroke index. The tension time index per minute rose less than with exercise, because of the smaller increase in the systemic systolic pressure. The left ventricular $\mathrm{dp} / \mathrm{dt}$ rose 43 per cent, due presumably to an increase in 
TABLE IVA

AVERAGE CHANGES OF HAEMODYNAMIC PARAMETERS BEFORE AND AFTER PHENTOLAMINE IN PATIENTS 1-5 WITH RELATIVELY NORMAL LEFT VENTRICLES

\begin{tabular}{|c|c|c|c|c|c|}
\hline & Rest & $\begin{array}{l}10 \text { min. } \\
\text { Phentolamine }\end{array}$ & $\%$ Change & $\begin{array}{l}20 \text { min. } \\
\text { Phentolamine }\end{array}$ & $\%$ Change \\
\hline 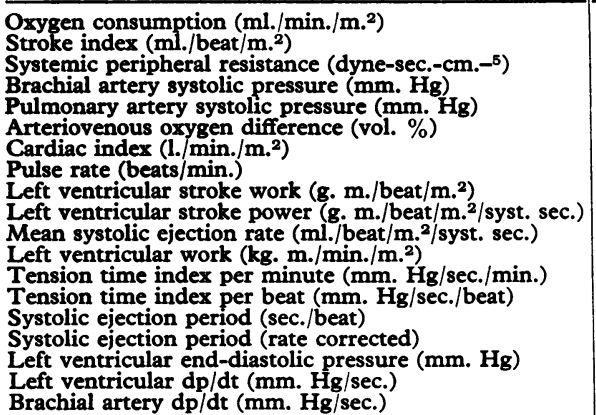 & $\begin{array}{l}137 \\
48 \\
1265 \\
127 \\
42 \\
4 \cdot 3 \\
3 \cdot 27 \\
71 \\
82 \\
287 \\
170 \\
4 \cdot 3 \\
2432 \\
34 \\
0 \cdot 28 \\
0 \cdot 40 \\
6 \\
633\end{array}$ & $\begin{array}{c}134 \\
48 \\
1347 \\
135 \\
43 \\
4 \cdot 1 \\
3 \cdot 68 \\
78 \\
88 \\
310 \\
171 \\
5 \cdot 2 \\
2878 \\
37 \\
0 \cdot 28 \\
0 \cdot 41 \\
5 \\
754\end{array}$ & $\begin{array}{r}-2 \\
0 \\
+7 \\
+6 \\
+2 \\
-5 \\
+13 \\
+10 \\
+6 \\
+8 \\
0 \\
+22 \\
+18 \\
+9 \\
0 \\
+2 \\
-17 \\
+48 \\
+19\end{array}$ & $\begin{array}{c}133 \\
43 \\
1522 \\
125 \\
47 \\
4 \cdot 3 \\
3 \cdot 20 \\
80 \\
73 \\
261 \\
152 \\
4 \cdot 3 \\
2712 \\
35 \\
0 \cdot 27 \\
0 \cdot 40 \\
4 \\
747\end{array}$ & $\begin{array}{r}-3 \\
-10 \\
+20 \\
-2 \\
+12 \\
0 \\
-2 \\
+13 \\
-11 \\
-9 \\
-10 \\
0 \\
+11 \\
+3 \\
-4 \\
0 \\
-33 \\
+58 \\
+18\end{array}$ \\
\hline
\end{tabular}

TABLE IVB

AVERAGE CHANGES OF HAEMODYNAMIC PARAMETERS BEFORE AND AFTER EXERCISE AND BEFORE AND AFTER PHENTOLAMINE IN PATIENTS 6-18 WITH ABNORMAL LEFT VENTRICLES

\begin{tabular}{|c|c|c|c|c|c|c|}
\hline & Rest & Exercise & $\%$ Change & Rest & $\begin{array}{l}20 \text { min. } \\
\text { Phentolamine }\end{array}$ & $\%$ Change \\
\hline 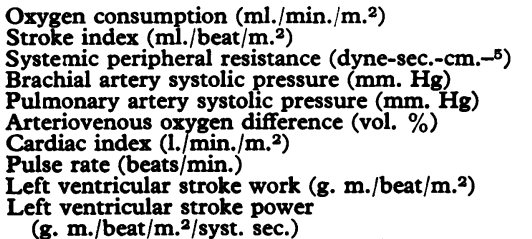 & $\begin{array}{r}123 \\
29 \\
1876 \\
137 \\
37 \\
5 \cdot 5 \\
2 \cdot 33 \\
82 \\
50 \\
198\end{array}$ & $\begin{array}{c}353 \\
37 \\
1419 \\
171 \\
75 \\
9 \cdot 4 \\
3 \cdot 83 \\
109 \\
79 \\
298\end{array}$ & $\begin{array}{r}+187 \\
+28 \\
-24 \\
+25 \\
+100 \\
+74 \\
+64 \\
+33 \\
+58 \\
+51\end{array}$ & $\begin{array}{c}138 \\
31 \\
1802 \\
139 \\
39 \\
5 \cdot 6 \\
2 \cdot 52 \\
84 \\
57 \\
205\end{array}$ & $\begin{array}{c}169 \\
42 \\
1364 \\
151 \\
35 \\
4 \cdot 8 \\
3 \cdot 64 \\
94 \\
84 \\
321\end{array}$ & $\begin{array}{r}+23 \\
+35 \\
-24 \\
+9 \\
-10 \\
-14 \\
+44 \\
+12 \\
+47 \\
+57\end{array}$ \\
\hline $\begin{array}{l}\text { Mean systolic ejection rate }\left(\mathrm{ml} . / \mathrm{beat} / \mathrm{m}^{2} / \mathrm{syst}^{\mathrm{sec}} \text { ) }\right. \\
\left.\text { Left ventricular work (kg. } \mathrm{m} . / \mathrm{min} . / \mathrm{m}^{2}\right) \\
\text { Tension time index per minute } \\
\text { (mm. Hg/sec./min.) }\end{array}$ & $\begin{array}{c}114 \\
3 \cdot 4 \\
2766\end{array}$ & $\begin{array}{c}150 \\
6.6 \\
4697\end{array}$ & $\begin{array}{l}+32 \\
+94 \\
+70\end{array}$ & $\begin{array}{c}116 \\
3 \cdot 7 \\
2950\end{array}$ & $\begin{array}{c}165 \\
5.6 \\
3283\end{array}$ & $\begin{array}{l}+42 \\
+51 \\
+11\end{array}$ \\
\hline $\begin{array}{l}\text { Tension time index per beat (mm. Hg/sec./beat) } \\
\text { Systolic ejection period (sec./beat) } \\
\text { Systolic ejection period (rate corrected) } \\
\text { Left ventricular end-diastolic pressure (mm. Hg) } \\
\text { Left ventricular dp/dt (mm. Hg/sec.) } \\
\text { Brachial artery dp/dt (mm. Hg/sec.) }\end{array}$ & $\begin{array}{l}34 \\
0 \cdot 25 \\
0 \cdot 38 \\
14 \\
734\end{array}$ & $\begin{array}{l}44 \\
0 \cdot 26 \\
0 \cdot 43 \\
25 \\
1151\end{array}$ & $\begin{array}{r}+29 \\
+4 \\
+13 \\
+79 \\
+81 \\
+57\end{array}$ & $\begin{array}{l}37 \\
0 \cdot 26 \\
0 \cdot 39 \\
14 \\
709\end{array}$ & $\begin{array}{l}37 \\
0 \cdot 25 \\
0 \cdot 39 \\
8\end{array}$ & $\begin{array}{r}0 \\
-4 \\
0 \\
-43 \\
+43 \\
+22\end{array}$ \\
\hline
\end{tabular}

left ventricular contractility. The left ventricular end-diastolic pressure fell 43 per cent. The left ventricular end-diastolic volume decreased in all subjects in whom this measurement was made. An increase in the systolic ejection fraction was also observed in all patients in Group 2.

No patient complained of any symptoms due to the infusion of the drug. No arrhythmias were noted and no patient developed chest pain. However, all the patients in Group 2 developed a desire to urinate during the infusion of the drug. A copious diuresis occurred at approximately 20 minutes, just before the second left ventricular angiogram.

\section{Discussion}

Taylor and his group (1965b, c) observed, after the administration of large doses of phentolamine, an increase in the cardiac output and heart rate and a fall in the systemic vascular resistance. They postulated that the decrease in the peripheral resistance produced a reflex increase in the cardiac output. Evans, Smulyan, and Eich (1967) recently observed a similar increase in the cardiac output after phentolamine administration, and came to a similar conclusion. Measurements of the force of contraction using a strain gauge arch, or of the speed of contraction, employing the left ventricular dp/dt, were not, however, recorded by these workers. Therefore, they could not evaluate any possible primary cardiac effect of the drug.

In our animal studies, the increase in the force of contraction after phentolamine injection suggested that this agent could improve cardiac dynamics in the Group 2 patients. By using the left ventricular $\mathrm{dp} / \mathrm{dt}$, we hoped to demonstrate that phentolamine 
had a positive inotropic effect in man. However, the left ventricular maximal $\mathrm{dp} / \mathrm{dt}$ increases with an acute increase in the heart rate, left ventricular end-diastolic pressure, mean aortic pressure, and aortic diastolic pressure, as well as with increased left ventricular contractility (Gleason and Braunwald, 1962; Wallace, Skinner, and Mitchell, 1963). All these parameters must, therefore, be analysed before an increase in contractility can be invoked. The determination of directional changes of the left ventricular first derivative can be accomplished with great accuracy using a catheter manometer system (Murphy et al., 1964). Thus, if the left ventricular $\mathrm{dp} / \mathrm{dt}$ shows a large percentage increase when all the other haemodynamic parameters remain essentially unchanged, then an increase in contractility must have occurred. In our Group 1 patients, the administration of phentolamine produced little or no change in the heart rate, cardiac pressures, cardiac output, or left ventricular volume. The 58 per cent increase in the left ventricular first derivative must, therefore, indicate an increase in contractility. In our Group 2 patients, the cardiac output rose significantly, not only because the heart rate was increased, but also because the stroke volume was augmented. The increase in the ejection of blood from the heart appears to be the result of positive inotropism and reduction in after-load to be due to contraction. The latter appears through primary vasodilatation and ease of run-off of blood during systole. The low dose of phentolamine (0.3 $\mathrm{mg}$./min.) that we employed in our study produced a rise in the systemic pressure due to the predominant inotropic effect. The higher doses used by other investigators probably produced predominant vasodilatation with a resultant fall in the systemic pressure, masking the inotropic effect of the drug. This is similar to the clinical experience with isoprenaline, which can initially produce a rise in systemic pressure due to the contractility effect. When the dose is increased, the systemic pressure falls, due to the dominant vasodilatation effect. This is not a new concept. Goodman and Gilman (1965) state that phentolamine given intravenously produces vasodilatation and cardiac stimulation. The blood pressure response varies with the relative contribution of the two effects. The dilatation is due primarily to a direct action on vascular smooth muscle, though with higher doses alpha-adrenergic blockade may also be involved. Our present work supports and extends their observations.

The conspicuous increase in the left ventricular $\mathrm{dp} / \mathrm{dt}$ in the Group 2 patients was consistent with an increased inotropism greater than that which could be attributed to cardiac rate. This was associated with a decrease in both the end-systolic and end- diastolic ventricular volumes and pressures. The reduced left ventricular end-diastolic pressure and the increased mean systolic ejection rate from a smaller end-diastolic volume can only result from an increased shortening velocity of the myocardial fibres (Sonnenblick et al., 1965), and thus reflects a positive inotropic action. The increased velocity of contraction is associated with an augmentation of myocardial oxygen consumption (Sonnenblick et al., 1965). The tension time index per beat (Sarnoff et al., 1958), which is another haemodynamic determinant of oxygen consumption of the heart, barely changed in our patients. However, with an increase in the heart rate, the tension time index per minute increased significantly.

The diuresis observed in this group of patients is probably due to the improvement in cardiac dynamics. The drug may also have a primary renal effect, but this was not investigated.

The marked increase in the cardiac output in the Group 2 patients and the negligible change in the cardiac output in the Group 1 patients may at first appear to be paradoxical. This varying response has, however, been explained by Braunwald, Ross, and Sonnenblick (1967). They state that the contractile state of the myocardium is not the factor that limits the volume of blood ejected by the heart in the normal person, and that an improvement of myocardial contractility by a drug with a positive inotropic effect, like digitalis, would not be expected to increase the output in a normal subject. On the other hand, in the presence of congestive heart failure, the cardiac output is usually limited by the contractile state of the myocardium, and a positive inotropic influence would be expected to raise the cardiac output (Braunwald et al., 1967). Similarly, the intravenous administration of a digitalis preparation to patients with mitral stenosis in sinus rhythm does not produce any essential clinical or haemodynamic improvement (Werkö et al., 1958; Beiser et al., 1968).

The positive inotropic action of phentolamine may be due to a direct or indirect mechanism. (1) Phentolamine may act like isoprenaline and directly stimulate the beta adrenergic sites in the heart; or (2) it may act like morphine and produce a sympatho-adrenal discharge which indirectly stimulates the beta-adrenergic receptors of the myocardium (Vassalle, 1961). Recently, Dairman and his associates (1968) have presented data that throw new light on phentolamine's mechanism of action. They administered phentolamine $(5 \mathrm{mg} . / \mathrm{kg}$.) to rats. At the height of receptor blockade, the conversion of a tracer dose of tyrosine $-{ }^{14} \mathrm{C}$ to norepinephrine in heart, brain, and adrenals was increased threefold, with no alteration in specific 
activity of tyrosine in blood and tissues. From these studies, they concluded that receptor blockade led to increased synthesis and release of norepinephrine in the 3 organs that were assayed. Our studies, with smaller doses of phentolamine, have indicated a positive inotropic effect, and in view of the findings of Dairman et al., it could reasonably be assumed that the inotropic effect is a direct one, resulting from increased production of catecholamines in the myocardium.

Clinical Implications. Several implications concerning the clinical management of patients in congestive heart failure or shock may be drawn from these findings. The beneficial effects of phentolamine are apparent. The patient in congestive heart failure shows a favourable haemodynamic response after the parenteral administration of phentolamine; this response will occur even if the patient is taking cardiac glycosides. In those patients with pulmonary oedema, phentolamine administration should produce a rapid alleviation of this cardiovascular emergency. It seems plausible that an effective oral form of this drug should also be of benefit for the patient in congestive heart failure.

Bradley and Weil (1965) have investigated the usefulness of phentolamine in 13 patients in shock. The drug was infused intravenously at an average rate of $0.6 \mathrm{mg}$. per minute for an average duration of 22 minutes. The cardiac output increased from an average of 2.8 to $3.61 . / \mathrm{min} . / \mathrm{m} .^{2}$ and the arterial pressure decreased from a mean of 73 to $59 \mathrm{mg}$. Hg. Improvement in systemic blood flow was manifested by a reduction in mean circulation time, an increase in peripheral skin temperature, and an increase in urine flow. They concluded that in selected instances an adrenolytic drug might be used in conjunction with, or in preference to, a pressor amine to improve effective blood flow in circulatory shock.

However, the decline in the arterial pressure limited the drug's clinical applicability for patients in shock. If one administers $0.3 \mathrm{mg}$. per minute of phentolamine to such patients, one might expect an increase rather than a fall in the systemic pressure. This is worthy of further investigation because of its clinical and therapeutic importance.

Since phentolamine has a positive inotropic effect, it will produce or increase the left ventricular gradient in patients with idiopathic hypertrophic subaortic stenosis. It may be preferable to use this agent rather than isoprenaline, since phentolamine does not produce an alarming increase in heart rate nor an alarming arrhythmia.
SUMMARY

In 4 dogs, the rapid intravenous injection of $5 \mathrm{mg}$. of phentolamine produced a 102 per cent average increase in the force of contraction, as measured by the strain gauge arch attached to the right ventricle.

After this experimental finding, 5 patients (Group 1) with relatively normal left ventricles who had never been in left ventricular failure, and 13 patients (Group 2) with abnormal left ventricles who had previously been in left ventricular failure, were studied at right and left heart catheterization. Cardiac pressures, cardiac output, and left ventricle $\mathrm{dp} / \mathrm{dt}$ were measured before, and 10 and 20 minutes after, the continuous intravenous administration of $0.3 \mathrm{mg}$. phentolamine per minute. The left ventricular end-diastolic volume was calculated angiographically before and after 25 minutes of continuous phentolamine administration.

Among the subjects in Group 1, phentolamine produced small varying directional responses in the cardiac index, with only minimal changes in the brachial artery systolic pressure and pulse rate. Among the subjects in Group 2, phentolamine produced a marked increase in the cardiac index with moderate increases in the brachial artery systolic pressure and pulse rate. The left ventricular $\mathrm{dp} / \mathrm{dt}$, which is a measure of contractility, increased in all patients. The left ventricular end-diastolic volume decreased in all subjects in whom this measurement was made.

It is concluded that phentolamine has a prominent inotropic effect. When administered to the patient in congestive heart failure, there is a striking haemodynamic improvement.

We are indebted to Dr. Hugh Butler and Dr. Peter Hofstra for placing the strain gauge arches on the dogs' ventricles.

\section{REFERENCES}

Ahlquist, R. P. (1948). A study of the adrenotropic receptors. Amer. F. Physiol., 153, 586.

Beiser, G. D., Epstein, S. E., Stampfer, M., Robinson, B., and Braunwald, E. (1968). Studies on digitalis. XVII. Effects of ouabain on the hemodynamic response to exercise in patients with mitral stenosis in normal sinus rhythm. New Engl. F. Med., 278, 131.

Bradley, E. C., and Weil, M. H. (1965). Treatment of circulatory shock with adrenolytic (vasodilator) drug. f. clin. Invest., 44, 1030.

Braunwald, E., Ross, J., Jr., and Sonnenblick, E. H. (1967). Mechanisms of contraction of the normal and failing heart. New Engl. F. Med., 277, 1012.

Dairman, W., Gordon, R., Spector, S., Sjoerdsma, A., and Udenfriend, S. (1968). Effect of alpha-blockers on catecholamine biosynthesis. Fed. Proc., 27, 240.

Davilla, J. C., and Sanmarco, M. E. (1966). An analysis of the fit of mathematical models applicable to the measurement of left ventricular volume. Amer. F. Cardiol., 18, 31. 
Dodge, H. T., Sandler, H., Ballew, D. W., and Lord, J. D. (1960). Use of biplane angiocardiography for the measurement of left ventricular volume in man. Amer. Heart F., 60, 762.

Evans, G. L., Smulyan, H., and Eich, R. H. (1967). Role of peripheral resistance in the control of cardiac output. Amer. F. Cardiol., 20, 216.

Gleason, W. L., and Braunwald, E. (1962). Studies on the first derivative of the ventricular pressure pulse in man. f. clin. Invest., 41, 80.

Goodman, L. S., and Gilman, A. (1965). The Pharmacological Basis of Therapeutics, 3rd ed., p. 557. Macmillan, New York and London.

Klein, M. D., Herman, M. V., and Gorlin, R. (1967). A hemodynamic study of left ventricular aneurysm. Circulation, 35, 614.

Murphy, G. W., Schreiner, B. F., Bleakley, P. L., and Yu, P. N. (1964). Left ventricular performance following digitalization in patients with and without heart failure. Circulation, 30, 358.

Sarnoff, S. J., Braunwald, E., Welch, G. H., Case, R. B., Stainsby, W. N., and Macruz, R. (1958). Hemodynamic determinants of oxygen consumption of the heart with special reference to the tension-time index. Amer. F. Physiol., 192, 148.

Sonnenblick, E. H., Ross, J., Jr., Covell, J. W., Kaiser, G. A., and Braunwald, E. (1965). Velocity of contraction as a determinant of myocardial oxygen consumption. Amer. F. Physiol., 209, 919.

Taylor, S. H., MacKenzie, G. J., George, M., and McDonald, A. (1965a). Effects of adrenergic blockage on the pulmonary circulation in man. Brit. Heart f., 27, 627.

-, Sutherland, G. R., MacKenzie, G. J., Staunton, H. P., and Donald, K. W. (1965b). The circulatory effects of intravenous phentolamine in man. Circulation, 31, 741. $\longrightarrow,-,-$, and $-(1965 \mathrm{c})$. The circulatory effects of phentolamine in man with particular respect to changes in forearm blood flow. Clin. Sci., 28, 265.

Vassalle, M. (1961). Role of catecholamine release in morphine hyperglycemia. Amer. F. Physiol., 200, 530.

Wallace, A. G., Skinner, N. S., Jr., and Mitchell, J. H. (1963). Hemodynamic determinants of the maximal rate of rise of left ventricular pressure. Amer. F. Physiol., 205, 30.

Werkö, L., Eliasch, H., Thomasson, B., and Varnauskas, E. (1958). Effect of acute digitalization in patients with rheumatic heart disease. Cardiologia (Basel), 33, 161. 